$\mathrm{XV}$.

\title{
Zur Casuistik der multiplen Hirnnervenlähmung durch Geschwulstbildung an der Schädelbasis.
}

Aus der medicinischen Klinik des Herrn Prof. Erb in Heidelberg.

\author{
Von \\ Dr. M. Dinkler, \\ I. Assistent der Klinik und Privatdocent.
}

Der bemerkenswerthe Aufschwung, den die Hirnchirurgie in den letzten Jahren durch das Verdienst Horsley's und v. Bergmann's genommen hat, macht es wahrscheinlich, dass mit der weiteren Ausbildung der Operationsmethoden auch andere Partien des Gehirns, als der Hemisphärenmantel, chirurgischen Eingriffen zugängig gemacht werden; so dürften vielleicht nach Vervollkommnung der manuellen und instrumentellen Technik unter dem Schutze der Antiseptik die krankhaften Veränderungen an der Schädelbasis, soweit sie in der vorderen Schädelgrube oder den seitlichen Theilen der mittleren und hinteren Grube liegen, auf blutigem Wege, sei es nun durch die Orbita oder die squamösen Abschnitte der Schläfenund Hinterhauptsbeine hindurch, behandelt und gelegentlich geheilt werden! Die wichtigste Vorbedingung für derartige verantwortliche Eingriffe ist zweifellos die sorgfältige Erwägnng der Diagnose, zu deren Stellung ein Zusammengehen und Zusammenarbeiten des Chirurgen von Fach mit dem internen Collegen ein absolutes Erforderniss ist und bleiben wird. Von der weiteren Entwicklung der Diagnostik der intracraniellen Affectionen, deren Endziel darin bestehen muss, die operablen Fälle möglichst frühzeitig zu erkennen und von den nicht operablen oder medicamentös zu beseitigenden zu trennen, wird es abhängen, ob die angedentete Möglichkeit, auch basale Erkrankungen operativ zu behandeln, ein pium desiderium bleiben wird, oder ob mit diesem Gebiet die chirurgische Kunst um ein weiteres Feld der Thätigkeit bereichert werden wird. - Wegen seines diagnostischen Interesses, wie auch wegen der genauen Beob- 
achtung des klinischen Verlanfes und der Bestätigung der Einzelsymptome durch die Nekropsie erscheint die Mittheilung folgenden Falles von Basaltumor berechtigt.

Adam Seip, 53jähriger Gastwirth von Heddesbach, wurde am 4. Februar 1889 in die medicinische Klinik aufgenommen.

Die damals erhobene Anamnese ergab Folgendes:

Eltern des Patienten (an Typhus, bezw. Wassersucht) gestorben, 3 Geschwister leben, sind gesund; kein ähnliches Leiden wie das des Patienten in der Ascendenz beobachtet. Patient selbst hat im 8. Jahre einen schweren Typhus und im 20. Jahre eine Lungenentzündung überstanden; seitdem stets gesund und arbeitsfähig, erkrankte er im September 1888, nachdem er sich angeblich 8 Tage vorher beim Arbeiten in einer offenen Scheune eine Erkältung zugezogen hatte, plötzlich an heftigen Kopfschmerzen, namentlich in der linken Hälfte des Hinterkopfs, die vor Allem bei horizontaler Lage (im Bett) beträchtlich an Intensität zunahmen. Weiterhin trat Doppels ehen hinza, die Sehschärfe blieb dabei anscheinend normal. Kein Erbrechen, kein Schwindel, keine Gehstörungen. Im October wurde in der hiesigen Augenklinik eine Parese des M. rectus internus und superior dexter bei ophthalmoskopisch normalem Befunde constatirt. - Da unter Gebrauch von Jodkali Schwindel und Ohrensausen und eher eine Verschlimmerung der ersten Erscheinungen eintrat, so liess sich Patient anderweitig behandeln. Erst im Januar 1889 stellte er sich wegen heftigen Drucks über dem rechten Auge und stechender Schmerzen im Innern desselben wieder vor und bat um Aufnahme in die Klinik. Nach abermaliger Jodkalimedication entstanden schon nach geringen Dosen so heftige Erscheinungen von Jodismus, dass das Mittel wieder ausgesetzt werden musste; dagegen brachte die Galvanisation des Kopfes in der Längsrichtung meist für den ganzen Tag Linderung der Schmerzen. Am 4. Februar 1889 trat Patient dann, da keine Besserung bemerkbar wurde, in die medicinische Klinik über.

Status praesens. Mittelgrosser, blasser Mann mit leidenden, deprimirten Zügen. Innere Organ e anscheinend frei von Veränderungen.

Nervensystem. Die meisten Gehirnnerven, insbesondere Hypoglossus, Glossopharyngeus, Acusticus, Olfactorius u. s. w. functioniren normal, während von Seiten des rechten Oculomotorius eine deutliche $P$ arese des $Z_{w}$ eiges für den Rectus internus und in geringerem Grade des Rectus superior besteht; Pupillen beiderseits gleichweit, reagiren accomodativ und reflectorisch normal.

Sensibilität und Motilität unverändert, Reflexe desgleichen; keine trophischen Störungen, keine von Seiten der Sphinkteren.

Urin albumin- und zuckerfrei; kein Fieber. Körpergewicht 50,7 Kgrm.

Aus den Aufzeichnungen uber den weiteren Verlauf sei Folgendes erwähnt:

8. Februar. Schmerzen im Kopf, besonders im rechten Hinterkopf und um das rechte Ange herum sehr heftig. (Ordin.: Jod und Brom, Galvanisation, Eisbeutel.) 
18. Februar. Conjunctivitis dextra; Conjunctiva bulbi dextri a uf Berührung ziemlich unempfindlich; das Auge wird meist geschlossen gehalten (reflectorisch wegen der Schmerzen im Inneren?); Schwindel and Ohrensausen treten regelmässig bald nach dem Aufstehen ein; Ohrensausen vorwiegend auf der rechten Seite.

25. Februar. Conjunctivitis geringer; Sensationen in der reehten Nasenhöhle; zunehmende Abmagerung und Blässe.

1. März. Schmerzen etwas gemildert; Conjunctivitis unverändert; ophthalmoskopisch auf dem rechten AugeErscheinungen von Papillitis. Das Zusammentreffen der bisher beobachteten Oculomotoriusund Trigeminus-(Ramus primus)Symptome mit den Veränderungen am rechten Augenhintergrund machen es bei dem Fehlen anderer Herdsymptome wahrscheinlich, dass es sich um eine retrobulbäre Affection mit neuroparalytischer Keratitis handelt.

14. März. Zu den Schmerzen in der rechten Kopfhälfte und im rechten Auge gesellen sich solche im rechten Ohr; Bulbus rechts etwas stärker prominirend (geringer Exophthalmus), Parästhesien über dem rechten Supraorbitalrand.

19. März. Rhinoskopische Untersuchung von Prof: Jurasz: Rhinitis chronica mit Secretion eines dicken eiterigen Schleimes, eitriger Katarrh des Cavam pharyngo-nasale; in der rechten Choane, seitlich von der mittleren Muschel, kirschkerngrosser, etwas transparenter Tumor (Polyp); ausserdem besteht im Kehlkopf eine Parese der $\mathrm{Mm}$. thyreo-arytaenoidei interni.

31. März. Schmerzen im ganzen Kopf, Brennen im rechten Auge, Schmerzen und Steifheit im Nacken.

10. April. Schmerzen besonders heftig in der rechten Schläfe; Lähmung des M. rect. int. et super. dext. unverändert, Sehschärfe des rechten Auges anscheinend nicht verändert, keine Hemianopsie; keine sonstige Motilitäts- oder Sensibilitätsstörung.

15. April. Entfernung des Nasenpolypen; Gefühl, als ob dauernd ein Tropfen kalten Wassers auf das rechte obere Lid falle; Kriebeln im rechten inneren Augenwinkel und auf dem Nasenrücken und rechten Nasenflügel; ophthalmoskopisch keine Aenderung.

28. April. Gefühl, als ob in der Mundhöhle Alles entzündet wäre (objectiv nichts). Sausen im rechten Ohr mit Schwerhörig keit, Gesicht und Conjunctiven stark geröthet; Gewichtsabnahme $3,5 \mathrm{Kgrm}$.

5. Mai. Patient sehmeckt angeblich nichts mehr.

9. Mai. Schwindel, Ohrensausen rechts; das Kauen orzengt lebhafte Schmerzen im ganzen rechten Trigeminusgebiet; mimische Bewegungen der rechten Gesichtshälfte weniger ausdrucksvoll als links.

14. Mai. Parese des rechten Facialisgebietes in allen 3 Aesten, am stärksten im Mentalast; die Furchen der rechtsseitigen Stirnfalten viel weniger stark als links, Augenschluss rechts matt, die Oberlippe wird dagegen rechts noch gut gehoben, die Unterlippe beim Zeigen der Zähne kaum bewegt; beim Versuch zu pfeifen entweicht die Luft durch die rechte Mundspalte, die Contraction des Platysma ist rechts 
weniger kräftig als links; Gaumensegel normal beweglich, desgleichen ist beim Bewegen der Kopfhaut keine deutliche Differenz nachweisbar. Zungenbewegungen normal, keine fibrillären Zuckungen, keine Atrophie. Schmerzen in der rechten Zungenhälfte, Gefühl von Schwellung derselben, sowie der rechtsseitigen Mundschleimhaut. Tastempfindung rechts normal, Schmerz- und Temperatursinn im rechten Quintusgebiet herabgesetzt - Parese des M. obliquus inferior und Paralyse des Rectus externus dexter; rechte Pupilleweiter als die linke, Reaction beiderseits schwach, Sensibilität des rechten Bulbus stark herabgesetzt, Cornealreflex fehlt. Mechanische Erregbarkeit im rechten Facialisgebiet etwas erhöht im Vergleich zu links, deutlich und zweifellos erhöht im Mentalgebiet, Zuckungen nicht träge; - Hörschärfe rechts sehr stark vermindert, Kaumusculatur beiderseits kräftig, Geruch und Geschmack rechts abgeschwächt; Unterkieferreflex nichtauszulösen, Sehnen- und Hautreflexe sonst normal.

18. Juni. Facialis parese geht zurück, elektrisch lässt sich parti elle EaR im ganzen rechten Facialisgebiet nachweisen, am stärksten in den beiden unteren Aesten (Herabsetzung der indirecten faradischen und galvanischen Erregbarkeit, galvanische directe Muskelerregbarkeit erhöht, Zuckungen träge, wurmförmig, AnSZ $>$ KaSZ, directe faradische Erregbarkeit erhalten aber herabgesetzt, Trägheit der Contraction). Sensibilität im rechten Trigeminus abnehmend, Trübung der Cornea bulbi dextri stärker. Ophthalmoskopischer Befund am linken Auge normal, am rechten Auge erscheint die Papille stark getrübt, geröthet, verschwommen, jedoch nicht prominent, die Gefässe sind gefüllt, jedoch nicht strotzeud, noch sind sie geschlängelt; ein kleiner Theil der Trübung der Papille ist wohl durch die Veränderungen der Cornea bedingt.

Diagnose: Neuritis n. optici dextri, keine Staungspapille (Dr. Bernheimer, Privatdocent).

25. Juni. Kopfschmerzen häufig so heftig, dass Patient laut a ufschreit. Mit dem rechten Ohr wird selbst die angelegte Uhr nicht mehr gehört, links auch nur auf $1 \mathrm{Cm}$. Entfernung. Fibrilläre Zuckungen im rechten Facialisgebiet, besonders in dem unteren und mittleren Ast, desgleichen in der Gegend der Mm. masseteres fibrilläre Unruhe, der rechte Masseter scheint schwächer zu sein als der linke. Geruchssinn rechts etwas vermindert, Geschmack scheint besonders auf der rechten vorderen $Z$ ungenpartie herabgesetzt zu sein.

1. Juli. Sehnen- und Hautreflexe der unteren Extremitäten, Bauchreflexe beiderseits normal; Schmerzen im rechten Quintusgebiet wechselnd (Augenwinkel, Stirn, Schläfe u. s. w.), seit kurzer Zeit auch Schmerzen im linken Supra- und Infraorbitalgebiet.

19. Juli. Otitis media purulenta sin., Perforatio membran. tympan.; keine Tuberkelbacillen im Secret. Parese des M. rect. infer. und intern. dext. geringer; psychisches Verhalten abnorm: Patient weint viel, spricht von Selbstmord, wird aggressiv gegen seine Mitkranken, weil sie schlecht von ihm sprechen. 
25. September. Zungenspitze weicht nach rechts ab beim Herausstrecken der Zunge; $\mathrm{Pupillarreaction} \mathrm{rechts} \mathrm{reflectorisch}$ undeutlich, accomodativ deutlich vorhanden, rechte Pupille mydriatisch. Parese des M. rectus extern. und super. sin.; Sensibilität im linken Supraorbitalgebiet herabgesetzt.

31. October. Parese der Kaumuskeln zu fast completer Paralyse gesteigert, Schlucken geht schlecht, rechte Facialisparese stärker, fibrilläre Zuckungen im rechten Facialis- und Quintus- (N. crotaphiticus)gebiet; Anästhesie im Gebiet des rechten Trigeminus; heftige Schmerzen und Parästhesien in der Schläfen- und Stirngegend.

12. November. Befallen sind:

1. N. olfactorius (functionirt beiderseits noch gering).

2. N. opticus beiderseits (links?).

3. N. oculomotorius (dexter in allen $\mathrm{Zweigen} \mathrm{complet} \mathrm{gelähmt,} \mathrm{Pu}$ pille starr, reactionslos.; N. oculomotorius sin. functionirt normal).

4. N. trochlearis dexter gelähmt. sin. normal.

5. N. trigeminus dexter gelähmt, Sensibilität aufgehoben, Cornealreflex fehlt, motorische Portion gleichfalls paralytisch (Atrophie des Masseter, mechanische Muskelerregbarkeit träge, Herabhängen des Unterkiefers, Hebung desselben unmöglich). - N. trigeminus sin. partiell gelähmt (Anästhesie im Gebiet des N. supraorbitalis, Verringerung der Sensibilität im 2. und 3. Ast; motorische Portion gelähmt).

6. N. abducens dexter et sin. gelähmt, letzterer nur paretisch.

7. N. facialis dexter paretisch mit partieller EaR, N. facialis sin. normal(?).

8. N. acusticus dexter völlige Taubheit; N. acust. sin. hochgradige Schwerhörigkeit (Otitis media!).

9. N. glossopharyngeus: (Süss, Sauer, Salzig, Bitter wird ziemlich richtig angegeben).

10. N. vagus (Puls klein, Herzaction unregelmässig, Frequenz 132 in der Minute).

11 und 12. Nn. accessor. und hypoglossus (wenig verändert, rechts etwas schwächer als links).

Sehnenreflexe und Hautreflexe schwach, aber deutlich auszulösen, Sensibilität und Motilität am übrigen Körper intact, mechanische Muskelerregbarkeit bei hochgradiger Abmagerung gesteigert, Sphinkteren normal; Lungen frei, Herztöne rein, Abdomen eingesunken, Leber und Milz bieten Nichts Abnormes. - Letztes Gewicht 40,9 Kgrm. (gegen 50,7 bei der Aufnahme).

23. December. Keine wesentliche Aenderung; unter den Erscheinungen hochgradigen Kräfteverfalls Exitus letalis.

Section (ansgeführt von Herrn Dr. Ernst): Magere, bräunlich pigmentirte Leiche; dickes Schädeldach, links von der Pfeilnaht im Scheitelbein eine markstückgrosse, tiefe Usur von Pacchioni'schen Granulationen. Die Dura an der Innenfäche glatt, das Gehirn haftet in den mittleren Schädelgruben etwas inniger an und reisst beim Herausnehmen an der Oberfäche leicht ein; Seitenventrikel weit und voll klarer farbloser Flüssigkeit; einzelne Piastellen stark ödematös, so dass die Pia 
blasenartig abgehoben ist. In der Hypophysisgegend wölbt sich eine hell rosafarbene höckerige Tumormasse vor, die Configuration der Sella turcica und des Clivus ist verstrichen und undeutlich. In der Tiefe der linken mittleren Schädelgrube prominirt eine ähnliche Masse von der Grösse einer Erbse, die rechte mittlere Schädelgrube ist von dieser Geschwulstmasse vollständig ausgegossen. Die umgebende Dura mater ist hyperämisch und lässt sich an diesen Stellen nur mit Mühe ablösen. In die Gehirnsubstanz geht die Masse nirgends über. Der rechte Abducens geht allmählich in eine spindelförmige Anschwellung von derselben Farbe und Consistenz wie der Tumor über; letzterer ist eher hart als weich und brüchig, zeigt keine Tendenz zum Zerfall. An den übrigen Gehirnnerven sind Veränderungen makroskopisch nicht wahrzunehmen. Gehirn und Medulla oblongata bieten auf frontalen Schnitten keine Veränderungen dar.

Anatomische Diagnose: Tumor des Keilbeines; Verwachsung der Hirnrinde mit der Dura mater der mittleren Schädelgruben; Hydrocephalus internus et externus u. s. w.

Nach mehrmonatlicher Härtung in Müller'scher Flüssigkeit und $\mathrm{Al}-$ kohol lassen sich an der Schädelbasis noch folgende Einzelheiten über die Grösse und die Verbreitung des Tumors und seine Beziehungen zu den einzelnen Gehirnnerven feststellen. Zunächst bildet der ganze Keilbeinkörper mit der Hypophysis eine einheitliche solide Masse, die die bekannte Configuration dieser Partie des knöchernen Schädels nur in groben Zügen wiedergiebt; die normalen Vorsprünge, welche den Türkensattel in Form der Proc. clinoidei anter., med. und post. begrenzen, fehlen gänzlich, dafür zeigt die Oberfläche des ganzen Tumor eine Anzahl bis erbsengrosser, warziger Erhebungen, über welchen die in ihrer Continuität erhaltene Dura mater cerebralis stark verdünnt ist. Nach den Seiten zu geht der Tumor unmerklich in die Sinus cavernosi über, und zwar ist der rechte so vollständig in der Neubildung aufgegangen, dass eine Abgrenzung desselben von der Sella turcica und der Hypophysisgegend nnmöglich ist; der 3., 4. und 6. rechte Hirnnerv besitzen bei ihrem Eintritt in den Tumor durch die Duralöffnungen noch annähernd normales Volumen und Aussehen. Auf einem Frontalschnitt durch die Mitte der Sella turcica zeigt sich weiter, dass der Keilbeinkörper mit den Sinus sphenoidales, der rechte grosse Keilbeinflügel bis in die Gegend des Foramen ovale durch Tumormasse ersetzt und der Knochen in ausgiebigem Maasse zerstört ist; die einzelnen Schädelöffnungen an der Innen- wie Aussenseite der Schädelbasis im Bereiche der rechten mittleren Schädelgrube sind in der einwuchernden Geschwulstmasse verloren gegangen. Besonders weit erstreckt sich ferner die Geschwulstbildung unter Usurirung des Knochens nach hinten und reicht die Dura vorwölbend auf der vorderen oberen Fläche der Pars petrosa des Felsenbeins bis zum Jugum petrosum, auf der hinteren bis in die Gegend des Sinus transversus. Von den dem Felsenbein zugehörigen nervösen Gebilden ist das Ganglion Gasseri mit seinen austretenden 3 Aesten vollständig von dem Neoplasma durchwachsen, so dass weder das Ganglion, 
noch die einzelnen Aeste sich isolirt darstellen lassen; auf frontalen Schnitten durch diese Gegend sind letztere zum Theil dadurch noch zu bestimmen, dass sich aus der gleichmässigen Neubildungsmasse ovale und rundliche Ringe von derbfarbiger Consistenz herausheben. Der VII. und VIII. Hirnnerv ist gleichfalls von Tumor umwachsen, aber noch einigermaassen frei zu präpariren. Der Facialis zeigt distal von dem Ganglion geniculi, welches anscheinend nur wenig vergrössert ist trotz seiner Verwachsung mit der Neubildung, eine auffallende Einschnürung und Verdünnung, die offenbar anf den Druck der wucherenden Tumormasse gegen die theilweise erhaltene knöcherne Wand des Canalis Fallopiae zurückzuführen ist; am Acusticus lässt sich keine deutliche Veränderung nachweisen. Eine genauere Untersuchung der Veränderungen des inneren Ohres, der Schnecke, des Labyrinthes, der Canales semicirculares ist unterblieben. - Von der Felsenbeinpyramide erstreckt sich in dünner Lage das Neoplasma noch über die vordere Hälfte der hinteren Schädelgrube und zeigt eine stärkere Wucherung im Bereiche des Foramen jugulare und des Canalis hypoglossi, deren Nerven gleichfalls vom Tumor umscheidet sind. Nach vorn erreicht der Tumor das Foramen opticum und führt durch die Verengerung desselben zu einer zwar geringen, aber deutlichen Atrophie des rechten N. opticus. Die Fissura orbitalis superior dextra ist völlig von dem Tumor austapezirt. Links von der Mittellinie geht er gleichfalls auf den Sinus cavernosus über, zeigt aber weit geringere Entfaltung als rechts, so dass die einzelnen nervösen Gebilde noch ziemlich gut zu isoliren sind; besonders ist das Ganglion Gasseri und der dritte Ast ergriffen. Ferner kriecht die Geschwulst auch links hinter dem Proc. clinoid. nach der hinteren Schädelgrube fort und bildet kleine Herde am Meatus auditorius internus und Foram. jugulare. Die hierdurch verlaufenden Hirnnerven sind ohne Schwierigkeit frei zu präpariren, das Ganglion geniculi erscheint dabei verdickt, sonst scheinen keine Veränderungen vorhanden. An den übrigen Theilen der Schädelbasis sind unter und in der abgelösten Dura keine Geschwulsteinlagerungen nachweisbar.

Die nur auf die Hirnnerven ausgedehnte mikroskopische Untersuchung - die Medulla oblongata und das Gehirn sind leider nicht aufbewahrt worden - ergiebt Folgendes:

[1. N. olfactorius beiderseits nicht untersucht.]

2. N. opticus dexter, der vor dem Herausnehmen unterbunden war, zeigt auf Querschnitten eine geringe, über die ganze Länge des Stammes bis zum Foramen opticum des Bulbus ziemlich gleichmässig entwickelte Erweiterung des intervaginalen Raumes; sowohl der subdurale, wie der subarachnoideale Theil enthält ein bald mehr, bald weniger reichliches zelliges Exsudat, dessen Einzelelemente den Charakter indifferenter Rundzellen tragen. An zahlreichen Stellen durchsetzen diese neugebildeten Zellen in continuirlichem Zuge die Pialscheide und verschmelzen mit gleichartigen Zellengruppen, die sich in den peripheren Nervenfaserbündeln vorfinden. Entsprechend diesen Zellwucherungen zeigen die Nervenfasern in der ganzen Peripherie des Opticus bald stärkere, bald geringere Veränderungen; schon mit einfacher Hämatoxylin- oder Boraxcarminfärbung, noch besser aber mit der W eigert'schen Methode lässt sich um den Opticus herum eine Art von Degenerationsring nachweisen; 
die destructiven Vorgänge vollziehen sich zunächst an den Markscheiden, welche zum Theil sehr beträchtlich aufquellen und schliesslich unter körnigem Zerfall gänzlich untergehen. Im Anschluss hieran geht auch der Axencylinder an Stellen intensiverer Veränderungen zu Grunde, so dass von einem derartig veränderten Faserbündel, welches durch das Pialseptum als selbständiger Complex abgeschlossen wird, nur das deutlich gxanulirte feinmaschige Gerüst der Neuroglia zurückbleibt. Von Tumormasse ist weder im Opticus selbst, noch im intervaginalen Raum etwas nachweisbar. Die in der Duralscheide verlaufenden $\mathrm{Nn}$. ciliares sind beträchtlich verändert, die Markscheiden sind völlig zerfallen, Schollen und Körner von Myelin erfüllen die Schwann'sche Scheide; auch die Axencylinder sind zum Theil zwischen den Zerfallsproducten nicht mehr vorhanden. Das Ganglion ciliare zeigt ausser einer mässigen Verdickung der die Ganglienzellen umgebenden Bindegewebsscheiden keine Anomalie.

Der linke N. opticus zeigt die eben geschilderten Veränderungen in weit geringerem Maasse.

3. N. oculomotorius dexter zeigt vor seinem Eintritt in den den Sinus cavernosus erfüllenden Tumor ebenso wie der $\mathbf{N}$. trochlearis und abducens normales Volumen. Mikroskopisch ist an diesem Theil die Pialscheide vollständig von der Geschwulst durchwachsen und mit der peripheren Zone des Nerven, welche gleichfalls von dem Neoplasma infiltrirt ist, so continuirlich verbunden, dass eine Trennung nicht möglich ist. Von diesem Geschwulstring, dessen Durchmesser mit der Annäherung an die Dura des Sinus cavernosus an Grösse auf Kosten des Nervenstammes wächst, gehen entsprechend dem Verlauf der von der Pia in den Nerven eindringenden Septa breitere und schmälere Zellenzüge hinein und bilden so innerhalb der die Peripherie einnehmenden Geschwulsthülle ein Netzwerk specifischer Zellenstrassen, deren Anordnung an den bekannten Typus der plexiform sich verbreitenden Gesehwülste erinnert. Zwischen dem auf diese Weise entstandenen Gerüst von Geschwulstmasse liegen die Reste der einzelnen Nervenfaserbündel, deren bindegewebiger Theil noch vielfach die Structur, wie man sie in normalen Nerven findet, erkennen lässt. Von dem Nervenparenchym selbst ist nichts erhalten, die Nervenfasern sind vollständig zerfallen und an ihrer Stelle finden sich zwischen dem offenbar erst secundär stark gewucherten Bindegewebe helle, meist kreisrunde Stellen, welche zum grösseren Theil wirkliche Hohlräume darstellen und nur an einigen wenigen Stellen in nach W eig e r gefärbten Präparaten bei starker Vergrösserung eine feinkörnige, blan tingirte Substanz nachweisen lassen. Ueber die Art und Weise, wie die Nervenfasern untergegangen sind, geben spärliche Gruppen von Myelinkugeln verschiedenster Grösse, welche mit Hämatoxylin gleichfalls noch die typische Kupferlack-Reaction geben, Aufschluss: zunächst sind offenbar die Markscheiden zu grösseren Schollen, diese dann durch weiteres Aufquellen zu grösseren und kleineren Myelintropfen und Kugeln zerfallen; in gleicher Weise ist der Axencylinder degenerirt und die beiderseitigen Zerfallsproducte sind zum Theil durch den Lymphstrom frei oder in Wanderzellen befindlich entfernt worden. Gerade die letzteren beanspruchen besonderes Interesse, weil ihr Vorkommen an Stellen, wo die Nervenstructur vollständig verwischt ist, über den ursprünglichen Verlauf 
und das Volumen des betreffenden Nerven oft den einzigen Anhaltspunkt darbietet; ihre Form ist bald oval, bald spindel- und sternförmig, je nach der Configuration der Gewebsspalten, in denen sie liegen; ausser einem ovalen Kern besitzen sie einen beträchtlichen Protoplasmaleib, der zahlreiche Myelinkngeln und Tropfen umschliesst und wegen dieses seines Inhaltes zu der Bezeichnung Körnchenzellen geführt hat. Behandelt man Präparate von gewissen Rückenmarkskrankheiten, bei denen die sogenannten Körnchenzellen regelmässig beobachtet werden, mit $\mathrm{der} W$ eigert'schen Färbung, so erhält man Bilder, mit denen die der eben beschriebenen Zellen vollkommen übereinstimmen. In der Anordnung der Körnchenzellen lässt sich eine gewisse Gesetzmässigkeit insofern enkennen, dass die dichtere Anhäufung immer in der Nähe der Pialscheide, in der die grösseren Lymphgefässe verlaufen, angetroffen wird. - Von Seiten des Bindegewebes ist, wie schon oben bemerkt, eine beträchtliche Hyperplasie und Kernwucherung hervorzuheben, die zum Theil den. Schwann'schen Scheiden, zum Theil den zwischen diesen verlaufenden Bindegewebssepten zufällt. Je weiter der Nerv in den Tumor selbst eindringt, um so mehr geht der charakteristische Bau des bindegewebigen Geriustes verloren und an seine Stelle tritt solide Tumormasse.

Der linke $N$. oculomotorius liegt dem von rechts her herüberwuchernden und in den Sinus cavernosus seine Ausläufer sendenden Tumor an, ohne jedoch direct in den neoplastischen Process mit einbezogen zu sein; mikroskopisch ist der Nerv nur wenig verändert, in einzelnen kleineren Bezirken zeigen sich Quellung und Auftreibung und beginnender Zerfall an den Markscheiden und umschriebene Kernwucherungen; Fortsetzungen der Neubildung in den Nervenstamm sind nicht nachweisbar.

4. Der $\mathrm{N}$. trochlearis dexter entspricht in seinem Verhalten dem des Oculomotorius dexter; er ist total degenerirt.

Der N. trochlearis sin. hingegen zeigt keine namhaften Veränderungen.

5. Der $\mathbf{N}$. trigeminus dexter ist vor dem Ganglion Gasseri ebenso wie der Oeulomotorius vor dem Eintritt in den Sinus cavernosus total degenerirt, alle Nervenfasern und Axencylinder sind zerfallen und an ihrer Stelle findet sich ein mehr oder weniger hyperplastisches Bindegewebe, vermischt mit verschieden breiten Zügen des Tumors. Das in die Nervenbahn der sensiblen Portio major des Trigeminusstammes eingeschobene Ganglion Gasseri ist bis auf einen kleinen Rest geschwunden; von Nervenfasern ist ausser den in spärlich vorhandenen Körnchenzellen eingelagerten Myelintheilen nichts mehr nachweisbar. Auch die Ganglienzellen sind fast vollkommen zerstört worden; unter den wenigen, die auf der Aussen- und Vorderfläche des Ganglions noch erhalten sind, besitzen nur einige normale Grösse, die meisten sind stark atrophirt, von polymorpher, bald ovaler, bald rundlicher oder birnförmiger Gestalt; an sämmtlichen fehlen die Fortsätze; die gleichfalls verschmächtigten Bindegewebsscheiden sind so eng an das Zellprotoplasma der Ganglienzellen angepresst, dass meist nur ihre scharf hervortretenden Kerne, welche die Protoplasmaperipherie der Ganglienzellen bis zu 3 und 4 an Zahl umsäumen, ihren Nachweis ermöglichen. Manche der Zellen, und zwar ausnahmslos die am meisten atrophischen, besitzen 'gar keine Scheide, sondern liegen wie 
kleine Inseln mitten in der Neubildung. Das Protoplasma ist an vielen sehr stark getrïbt und hat geradeza einen homogen bräunlichen Farbenton; andere Zellen sind auffallend blass, anstatt einer gleichmässigen protoplasmatischen Substanz enthalten sie innerhalb der atrophischen Bindegewebsscheide eine körnig-fadige kernlose Masse, die geronnener Lymphe sehr ähnlich sieht. Auch Spaltbildungen und Risse finden sich häufiger in den Zellen. Das Pigment bietet sowohl in der Menge, sowie in der Form gewisse Schwankungen, bald ist die ganze Zelle dicht erfülit von feinen Körnchen, die zu einer Art von Pigmentklumpen gelegentlich verschmelzen, bald bildet der Farbstoff nur eine kleine Sichel, die dem Kern haubenförmig anfsitzt. - In gleicher Weise wie das Ganglion Gasseri und die in dasselbe eintretenden Nerven sind auch die Nervenfasern der distal aus ihm sich bildenden drei Nervenstämme vollständig degenerirt; nur grössere Gruppen von Körnehenzellen vermögen die ursprüngliche Lagerung noch anzudeuten. - Der linke $N$. trigeminus zeigt im Vergleich zu dem rechten geringe Veränderungen; dieselben erstrecken sich vorwiegend auf den Ramus primus und die Portio minor and bestehen in degenerativen Erscheinungen an den Markschęiden. Die Axencylinder sind nur in der motorischen Partie des 3 . Astes zum grösseren Theil mit zerfallen. Als Ursache dieser Veränderungen ist einmal das Einwuchern des Tumors in das Epineurium und zweitens in die Nervenstämme selbst (in der schon früher beschriebenen Weise) zu erwähnen. Das Ganglion zeigt ausser einem Ausfall einer grösseren Zahl von Fasern keine besonderen Anomalien.

6. Der N. abducens dexter ist vollkommen zerstört; der linksseitige enthält eine nur kleine Anzahl erkrankter Fasern.

7. Der N. facialis dexter zeigt distal von dem Gangl. geniculi eine bedeutende Verschmälerung oder Einschnürung; mikroskopisch sind die Nervenfasern des centralen wie peripher davon gelegenen Stückes (bis zum Foramen stylomastoideum), die Markscheiden zum grössten Theil in grössere und kleinere Myelinschollen zerfallen, die Axencylinder scheinen bei Boraxcarminfärbung nicht verändert; nur an der Einschnürungsstelle ist ein Theil offenbar durch Druckatrophie in seiner Continuität unterbrochen, ein anderer zeigt Aufquellung und Verdickung. Das Ganglion genic. selbst ist zum grösseren Theil an der inneren Seite von Tumor durchsetzt und weist hierin ähnliche Veränderungen der Ganglienzellen auf, wie das Ganglion Gasseri. - Der N. facialis sin. lässt an den Nervenfasern nur in der Nähe des Ganglion geniculi eine unregelmässige Contourirung der Markscheiden und eine Aufquellung erkennen; kurz vor dem Austritt durch das Foramen stylomastoideum ist der Querschnitt wieder vollkommen normal; hingegen sind die Ganglienzellen des Ganglion geniculi zum grossen Theil zerstört, ebenso die zwischen ihnen verlaufenden Nervenfasern.

[8. N. acusticus sin. et dexter nicht untersucht.]

9. N. glossopharyngeus dexter zeigt beträchtliche Veränderungen der Nervenfasern. Die groben Fasern, welche ungefähr die Hälfte sämmtlicher betragen; haben eine Quellung der Markscheide und Zerfall in kleine Schollen erfahren; auch die Axencylinder sind zum Theil zerfallen. In ähnlicher Weise sind auch die feinen Fasern an der Degeneration betheiligt; die Kernvermehrung des Stützgewebes ist gering, nur umschrieben treten öfters kleinere Zellencomplexe hervor, die von den durch den Tumor 
gebildeten Zellenzügen nicht zu unterscheiden sind. Zweifellos neoplasmatischer Natur sind die Gewebstheile, die in der Nervenscheide sich entwickelt und so eine allseitige Compression des Nerven bewirkt haben. N. glossopharyngeus sin. enthält nur wenig veränderte Nervenfasern.

10 und 11 . N. vagus et aecessorius dexter et sinister entsprechen dem Bau des eben beschriebenen gleichseitigen Glossopharyngeus.

12. Im $\mathrm{N}$. hypoglossus dexter finden sich beträchtliche Veränderungen an den Markscheiden; das Myelin ist zerklüftet und zerfallen und zum Theil schon so vollständig resorbirt, dass im Innern der Schwann'schen Scheide nur der nackte Axencylinder nachweisbar ist. Der Tumor ist nicht auf den Nerven selbst übergegangen, sondern hat durch Druck von aussen, indem er den Eingang des Canalis hypoglossi ansfüllt, die destructiven Erscheinungen hervorgerufen.

Der N. hypoglossus sin. zeigt annähernd normales Verhalten.

Schliesslich erübrigt es noch auf den histologischen Bau des Tumors selbst und seine Entwicklung einzugehen. Der Ausgangspunkt ist bei der grossen Ausdehnung, die sich bei der Nekropsie herausstellte, nicht mit voller Sicherheit zu bestimmen, doch scheint der primäre Sitz einmal wegen der klinischen Initialsymptome von Seiten des rechten Oculomotorius und zweitens wegen der überwiegenden Verbreitung nach rechts (rechter Keilbeinflügel, rechtes Schläfenbein u. s. w.) mit hoher Wahrscheinlichkeit in den rechten Sinus cavernosus oder die rechte Hälfte des Keilbeinkörpers (cranielles oder Sinusperiost) verlegt werden zu müssen. Von der Hypophysis ist ein links von der Medianlinie liegender kleiner Abschnitt noch wohlerhalten und spricht gerade die scharfe Abgrenzung desselben gegen den Tumor gegen die Annahme, dass dieHypophysis selbst der Ausgangspunkt gewesen ist. Ihrem Ban nach ist die Neubildung ein Rundzellensarkom, dessen Zellenelemente es vermöge ihrer durchschnittlichen Grösse in die Gattung der kleinzelligen Rundzellensarkome einreihen. Der Reichthum an Blutgefässen zeigt in den einzelnen Abschnitten ziemlich beträchtliche Differenzen; am gefässreichsten sind zweifellos diejenigen Theile, welche zwischen den usurirten Knochenbälkehen angetroffen werden. Die Zerstörung des Knochens selbst ist zum Theil durch directe Einwirkung der Sarkomzellen, zum Theil auch durch die Thätigkeit von wohlausgebildeten Riesenzellen, sogenannten Osteoklasten, erfolgt, nnd zwar in der Weise, dass zunächst durch Ausziehen der Kalksalze der Knochen in osteoides Gewebe umgewandelt worden ist und dieses osteoide Gewebe eine fibröse Metamorphose und schliesslich einen vollständigen Zerfall erfahren hat. An den Gefässen selbst lassen sich ausser hyaliner Degeneration in beschränkter Ausdehnung keine Anomalien nachweisen; Blutungen von grösserem Umfang sind nicht vorhanden. Das Gewebe des Tumors zeigt nur im Bereich des rechten Sinus cavernosus, besonders im Centrum, stellenweise Neigung zu fettigem Zerfall, die Zellen werden opak, ihre Grenzen verwischt, Kern und Protoplasma gehen ineinander über and bilden eine an Fettkörnchen reiche, stark granulirte Detritusmasse. Innerhalb der Partien, welche in ursprünglich knöchernen Theilen liegen, scheint sich an der Resorption der Zerfallsproducte noch eine fibröse Wucherung 
und eine Entwicklung von Narbengewebe anzuschliessen. Bezüglich der Ausbreitung der Neubildung kann noch hinzugefügt werden, dass auch an der äusseren Fläche der Schädelbasis, vor Allem im Bereiche der rechten Tuba Eustachii, sarkomatöse Wucherungen von demselben Charakter wie im Sinus cavernosus nachweisbar sind. Die Carotis interna verläuft unverändert durch den Tumor hindurch.

Wenn wir den eben beschriebenen Fall noch einmal klinisch und anatomisch in seinen Hauptziugen recapituliren, so handelt es sich bei einem in keiner Weise hereditär belasteten 53jährigen Mann um die Entwicklung einer Hirnbasiserkrankung, für welche keines der bekannten Momente: Trauma, Syphilis und Tuberculose, von ätiologischer Bedeutung erscheint. Zu den ersten, gleichsam prodromalen Erscheinungen heftiger Schmerzen im Hinterkopf, die vor Allem im Liegen besonders intensiv auftreten, gesellen sich Doppeltsehen bei anscheinend normaler Sehschärfe und heftige neuralgische Empfindungen, sowie Parästhesien im Gebiet des Ramus primus des rechten Trigeminus (sowohl im Bereich der äusseren, die Stirn und den Schädel versorgenden.Zweige, als auch im Gebiete der nasalen und zum Augapfel herantretenden sensiblen Aestchen); aus der Neuralgie bildet sich rasch eine Anästhesie, und zwar unter Fortdauer der Schmerzen eine Anaesthesia dolorosa aus; weiterhin fällt von Seiten des Augapfels eine Protrusion aus der Orbita und eine

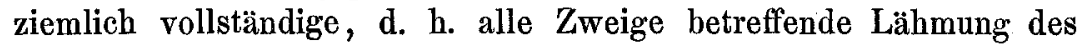
rechten Oculomotorius (mit Mydriasis und träger Pupillenreaction) und des Trochlearis und Abducens auf. Unter dem Eindruck der eben genannten Nervenlähmungs- und Reizerscheinungen, die ausgesprochene Herdsymptome genannt werden müssen, scheint die Localisation des Krankheitsherdes ohne Schwierigkeit, als nach kurzer Zeit der 2. und 3. Ast des rechten Trigeminus unter den gleichen Symptomen wie der 1. ergriffen werden und eine rechtsseitige Facialislähmung mit dem Charakter der peripherischen Form hinzutritt; wenige Tage nach der Entwicklung der letzteren lässt sich partielle EaR nachweisen und besteht längere Zeit unverändert fort. Auffallenderweise zeigt diese Lähmung im Gegensatz zu der progressiven Natur des Leidens deutliche Schwankungen: bald Besserung, bald Verschlimmerung, während die Herabsetzung der Hörschärfe bis zur fast vollständigen Taubheit fortschreitet und dann stabil bleibt und bemerkenswertherweise ophthalmoskopisch im rechten Auge eine Neuritis optica nachweisbar wird. Zu der Anästhesie im rechten Trigeminus treten weiterhin unter vereinzelten 
Anfällen von Erbrechen und starkem Schwindel (letzterer immer beim Aufrichten) psychische Störungen (Verfolgungsideen u. s. w.) und die Symptome einer motorischen Lähmung des 3. Astes des Trigeminus beiderseits, sowie sensible Störungen im ersten und zweiten Ast des linken Trigeminus (neuralgische Schmerzen, Herabsetzung der Sensibilität im linken Supraorbitalis). Auch die Zungenbewegungen scheinen eingeschränkt, wie die Deviation der Spitze nach rechts beim Herausstrecken der Zunge beweist. In diesem Zustand erfolgt unter dem Bilde allgemeiner Kachexie und hochgradiger Emaciation der Kräfte der Exitus letalis. Die anatomische Untersuchung bestätigt in jeder Richtung die klinische Beobachtung, denn es findet sich sowohl der rechte 2 . bis 12 . wie auch der linke 5. u. s. w. Hirnnerv mehr oder weniger hochgradig degenerirt. Angesichts der genannten Symptome steht die klinische Diagnose auf „basale Affection" ausser jedem Zweifel, ja es ist mit Rücksicht auf die Aufeinanderfolge der Einzelerscheinungen, sowie im Hinblick auf die Entwicklung und die Form der einzelnen Lähmungen (sensible Reizerscheinungen, peripherische Facialislähmung) auf der $\mathrm{Höh}$ e der Krankheit sogar leicht, nicht nur den Sitz genau zu präcisiren, sondern auch den Ausgangspunkt mit einer gewissen Sicherheit festzustellen und über die Natur der Erkrankung selbst die nöthige Klarheit zu gewinnen. Während es in einem der früheren Stadien des Processes bei dem Bestehen der Ophthalmoplegia externa et interna, der Protrusion des Bulbus, der Neuralgie und Anästhesie im ersten Ast des rechten Quintus nahe lag, eine retrobulbäre, in der Gegend der Fissura orbitalis superior und des Foramen opticum entstandene Neubildung als die Ursache des genannten Symptomencomplexes anzunehmen, wurde diese Wahrscheinlichkeitsdiagnose durch das Hinzutreten von Störungen im rechten Facialis und im $\dot{2}$. und 3. Ast des rechten, sowie 1., 2. und 3. Ast des linken Trigeminus unhaltbar. Denn vorausgesetzt, dass der primäre Sitz der Affection der hintere Theil der Orbita und der kleine Keilbeinflügel gewesen und von dort aus die Ausbreitung über die mittlere und hintere Schädelgrube erfolgt wäre, bliebe es doch schwer verständlich, dass ein Exophthalmus höheren Grades vermisst wurde, und dass vor Allem auch die links von der Medianlinie liegenden Nerven erkrankten, und zwar von diesen wiederum die besonders, welche von dem supponirten Ausgangspunkt des Processes möglichst weit entfernt liegen. Sehr wohl lässt sich hingegen die Entwicklung wie der ganze Verlauf dann erklären, wenn man den räumlichen Beginn der Erkrankung in den rechten Sinus cavernosus verlegt, wo der 1 . Ast des Trigeminus und 
der Oculomotorius, von denen beiden die Initialsymptome: die Diplopie einer- und die Neuralgie andererseits ausgegangen sind, bei einander liegen. Von dieser Centralstelle aus nach vorn und hinten, nach links und rechts sich verbreitend kann die Geschwulst die Neuritis optica unilateralis, die Ophthalmoplegie, die Lähmung des rechten und linken Trigeminus, die rechtsseitige Facialisparese in einer der klinischen Beobachtung entsprechenden Reihenfolge hervorrufen. - Bei der genaueren Bestimmung der Natur des Processes kommen zwei Krankheiten in Frage: Entzündung und Neoplasma. Von den entzündlichen Affectionen verdienen nur die chronisch progressiv verlaufenden: die tuberculösen und syphilitischen, Erwähnung; die ersteren von ihnen lassen sich, ganz abgesehen von dem Fehlen jeder hereditären Belastung oder manifester tuberculöser Processe am iubrigen Körper, wegen des fieberlosen Verlaufs, der eigenartig begrenzten, wenn schon bedeutenden Ausdehnung, der vorwiegend einseitigen Localisation und wegen des Fehlens schwererer Allgemeinerscheinungen während des grösseren Abschnittes der Krankheit mit ziemlicher Sicherheit ausschliessen; anch für eine syphilitische Meningitis oder Neuritis (analog der Wurzelneuritis) ist, abgesehen von dem vollständigen therapeutischen Misserfolg der Jodkalicuren, die Localisation, der Verlauf, die Intensität der basalen Symptome bei dem Mangel anderweitiger ernster Störungen, z. B. von Seiten der grauen Gehirnrinde, als höchst auffallend zu bezeichnen. Es bleibt demnach in Erwägung des progressiven Charakters und des fieberlosen Verlaufs der Krankheit nur die Annahme einer Neubildung im engeren Sinne übrig; da das fragliche Neoplasma maligner Natur sein muss, wie aus dem raschen Wachsthum, aus seiner Grösse und aus der totalen Zerstörung der meisten Nervenstämme hervorgeht, so können nur die Sarkome und Carcinome in Frage kommen. Die letzteren kommen intracraniell - von einem vom Pharynx, resp. dem Cavum pharyngonasale $\mathfrak{u}$.s. w. in die Schädelhöhle eingewucherten kann füglich keine Rede sein - nur höchst selten primär (von der Hypophysis?) vor, so dass man ohne Bedenken von ihnen abstrahiren und sich für die sarkomatöse Beschaffenheit entscheiden kann. Eine weitere Frage ist nach den tiber die Natur der Geschwulst angestellten Erörterungen noch die, hat die Dura mater des Sinus transversus dexter, die Gefässscheide der Carotis interna, die Hypophysis der Keilbeinkörper oder deren Sinus den eigentlichen Ausgangspunkt, den Mutterboden, abgegeben? Nach dem klinischen Verlauf scheint die Annahme von der Entstehung im Sinus cavernosus dexter am wahrscheinlichsten, weil dann bei Voraussetzung eines allseitig gleich- 
mässigen Wachsthums das frühere und vorwiegende Befallensein der rechtsseitigen Hirnnerven und das relativ späte Auftreten der gleichseitigen Neuritis optica u. s. w. eine ungezwungene Erklärung findet; gegen den Ausgang von der Hypophysis') spricht - abgesehen von dem früher erwähnten Verhalten des mikroskopisch nachgewiesenen Restes dieses Organs zum Tumor - das Fehlen von Chiasmaläsion, cerebraler Herd- und Allgemeinsymptome u. s. w. Zu einer ganz exacten Localisation des örtlichen Beginns vermag jedoch auch die anatomische Untersuchung nicht zu verhelfen, da die elementaren Bestandtheile des Tumors in diesem Sinne keine charakteristischen Eigenschaften besitzen; jedenfalls ist es wahrscheinlich, dass die Geschwulst nicht genau in der Medianlinie, sondern etwas nach rechts von derselben entstanden oder zum Mindesten nach rechts zu ganz unverhältnissmässig rasch gewachsen ist. Wie schwierig übrigens in manchen Fällen die Entscheidung äber die Natur und den Ausgangspunkt einer basalen Erkrankung sein kann, beweist der von Unverricht ${ }^{2}$ ) mitgetheilte Fall von multipler Hirnnervenlähmung; so sicher in dieser Beobachtung das Symptomenbild den Weg, auf welchem die Affection um sich griff, zeichnete, so unsicher waren doch alle Vermuthungen über die anatomische Beschaffenheit und die Localisation der Krankheit, bis die Autopsie post mortem jeden Zweifel durch den Nachweis eines primären Mediastinaltumors, von dem aus der diffuse basale Process als ein metastatischer ausgegangen war, beseitigte.

In der Literatur findet sich eine Anzahl ähnlicher Beobachtungen wie die vorliegende mitgetheilt und die Symptomatologie dieser Fälle hat schon unter anderen in den Lehrbüchern von Wernicke und Nothnagel eine eingehende und vortreffliche Würdigung erfahren. Wenn schon eine Beobachtung, wie die Romberg's ${ }^{3}$ ), in welcher es sich um eine Neuralgie des 1. linken Trigeminusastes infolge von Aneurysmabildung der Carot. intern. im Sinus cavernosus handelt, nach unseren bisherigen Erfahrungen wegen der geringen Ausbildung und des sich lange Zeit (18 Jahre) völlig gleichbleibenden Befunds nicht zu erkennen ist, so zeigen doch die Fälle von v. Zie mssen ${ }^{4}$ ), v. Graefe ${ }^{5}$ ),

1) vgl. W. Rath, Archiv für Ophthaimologie. Bd. XXXIV. 3. S. 105: Beitrag zur Symptomenlehre der Geschwülste der Hypophysis.

2) Multiple Hirnnervenlähmung. Fortschritte der Medicin. 1887. S. 791 u. ff.

3) Lehrbuch der Nervenkrankheiten. 3. Auflage 1883.

4) Virchow's Archiv. Bd. 13.

5) v. Graefe's Archiv. Bd. 12. Abth. II. S. 100. 
Bell') und $\mathrm{Hulke}^{2}$ ) und Anderen, dass das klinische Bild der im Bereich der mittleren Schädelgrube entstehenden Tumoren ziemlich hänfig charakteristisch genug ist, um nicht allein ihre Ausdehnung, sondern auch den ursprïnglichen Herd intra vitam zu bestimmen; allerdings ist es, wofür auch der von uns mitgetheilte Fall Zeugniss ablegt, von der früheren oder späteren Betheiligung der einzelnen Hirnnerven abhängig, ob eine richtige Analyse des Processes schon im Beginn oder erst auf der Höhe der Krankheit möglich ist. Von ausschlaggebender Bedeutung in diagnostischer Hinsicht scheinen mir folgende drei Momente:

1. das Verhalten der Trigemini,

2. das der Nn. optici, und

3. die Reihenfolge, in welcher die einzelnen Symptome auftreten.

Zunächst wird durch das Auftreten von Trigeminusneuralgien der peripherische Charakter einer intracraniellen Läsion absolut sicher gestellt, da sensible Reizerscheinungen - und eine andere Deutung der Neuralgien lässt sich nicht geben - nur bei peripherischen Veränderungen, nie bei nucleären oder cerebralen vorkommen. Mit diesem Nachweis des peripherischen Sitzes ist zugleich das Gebiet, innerhalb dessen die Erkrankung nur liegen kann, scharf und sicher abgegrenzt: entweder muss der Herd in der Nähe der Fissura orbitalis oder im hinteren Theil der mittleren Schädelgrube localisirt sein, je nach der isolirten Betheiligung des 1. Astes oder der vereinigten Läsion des 1. und 2. oder aller 3 Aeste. Besondere Vorsicht ist, wie der mitgetheilte Fall zeigt, in der diagnostischen Verwerthung einer alleinigen Erkrankung des 1. Astes deshalb geboten, weil der Process trotz der Entstehung im hinteren Theil der mittleren Schädelgrube im Beginn nur den ersten, nach längerem Freibleiben schliesslich auch unter entsprechender Ausdehnung den 2. und 3. Ast befallen kann. - Von ähnlichem diagnostischen Werth wie die Quintuserscheinungen ist die einseitige Opticuserkrankung. Während eine doppelseitige Neuritis oder Staungspapille nur für eine entzündliche Veränderung oder Raumbeengung im Allgemeinen zu verwerthen ist, hat die unilaterale Erkrankung des Opticus, gleichviel ob sie dauernd als solche besteht oder nach längerer Zeit noch von der des anderen Auges gefolgt ist, stets die Bedeutung eines

1) Physiolog. und pathol. Untersuchungen des Nervensystems. Uebersetzung von Romberg. 1832. S. 217.

2) Ophth. Hosp. Rep. VI. April 1868. 
Herdsymptoms; denn durch sie wird nicht nur die Seite des primären Krankheitssitzes bestimmt, sondern derselbe wird auch mit Bestimmtheit auf den medialen vorderen Theil der mittleren Schädelgrube centrirt. Einer besonderen Begrindung dieses Satzes bedarf es nicht, die anatomischen Verhältnisse geben gentigende Aufklärung. - Von nicht geringer Bedeutung für die Erkennung der Basalerkrankung ist schliesslich das dritte der oben genannten Momente: die zeitliche Aufeinanderfolge, die Gruppirung der einzelnen Symptome. Bei sorgfältiger Erwägung der Topographie vermag die Combination der klinischen Erscheinungen der Localisation einen hohen Grad von Sicherheit $\mathrm{zu}$ verleihen, vorausgesetzt, dass der Grundsatz immer festgehalten wird, alle Störungen wenn möglich durch einen einzigen Krankheitsherd zu erklären und diesen immer dahin zu localisiren, wo die einzelnen erkrankten Bahnen auf einem möglichst kleinen Bezirk vereinigt sind.

Unter Berïcksichtigung dieser Einzelregeln wird die genaue und frühzeitige Bestimmung des Sitzes basaler Erkrankungen in einer Anzahl von Fällen ohne grosse Schwierigkeiten gelingen und die eventuelle Therapie auf der breiten Grundlage einer exacten Diagnose sich feststellen lassen. 